\title{
Desarrollo de un modelo de gestión del conocimiento para las PYMES del sector textil de la ciudad de Riobamba
}

Resumen

Las PYMES en el Ecuador se encuentran en particular en la producción de bienes y servicios(ALADI 2001), siendo la base del desarrollo social del país tanto produciendo, demandando y comprando productos 0 añadiendo valor agregado, por lo que se constituyen en un actor fundamental en la generación de riqueza y empleo, en estudios previamente guiados por el Ministerio de Productividad (Equipo-Ecuador, financiado por el BID), estas organizaciones representan el $95 \%$ de las unidades productivas del país, generan el $60 \%$ del empleo, participan del $50 \%$ de la producción, tienen un amplio potencial redistributivo, importante capacidad para la generación de empleo, amplia capacidad de adaptación y flexibilidad frente a los cambios.

La investigación realizada diseña un Modelo de Gestión de Conocimiento para este importante sector de la economía nacional tomando como unidad de estudio la cadena de suministro de las PYMES del sector textil de la ciudad de Riobamba, sobre la base de dos variables, la Gestión del Conocimiento con tres dimensiones: Gestión Estratégica del Conocimiento, Ambiente Innovador y Ciclo del Conocimiento o Gestión Funcional de Conocimiento y una variable Dependiente que es la Percepción de Resultados del sistema ha sido definida a efectos del presente estudio, como la formulación de juicios que sobre la realidad tienen las personas que integran las organizaciones, utilizando en este caso como referencia el Cuadro de Mando Integra CMI en sus dimensiones innovación, mejora de procesos internos, relaciones con el entorno y económica, a las cuales se adicionó una comparación referencial con empresas y trabajadores con funciones similares.

Palabras Clave: Gestión del Conocimiento; PYMES Percepción de Resultados; Estrategia; Innovación; Ciclo de Conocimiento

DEVELOPMENT OF A KNOWLEDGE MANAGEMENT MODEL FOR SMALL AND MEDIUM-SIZED TEXTILE SECTOR IN THE CITY OF RIOBAMBA

Summary

SMEs in Ecuador are particularly involved in the production of goods and services (ALADI 2001), being the basis of the social development of the country both producing, demanding and buying products or adding added value, so that they constitute a fundamental actor These organizations represent $95 \%$ of the productive units of the country, generate $60 \%$ of the employment, participate in the $50 \%$ of production, have a large redistributive potential, important capacity for job creation, broad adaptability and flexibility in the face of change.

The research carried out designs a Knowledge Management Model for this important sector of the national economy, taking as a unit of study the supply chain of SMEs in the textile sector of the city of Riobamba, based on two variables, Knowledge Management With three dimensions: Strategic Management of Knowledge, Innovative Environment and Knowledge Cycle or Functional Management of Knowledge and a Dependent variable that is the Perception of Results of the system has been defined for the purposes of this study, as the formulation of judgments about reality In this case, the CMI Integral Control Panel in its dimensions of innovation, improvement of internal processes, relations with the environment and economic, to which was added a reference comparison with companies and workers with Functions.

Keywords: Knowledge Management; SMEs; Results Perception; Strategy; Innovation; Knowledge Cycle

\section{INTRODUCCIÓN}

En el entorno económico de los negocios en el Ecuador cuya economía es considera pequeña (Araque 2000), se demanda que las organizaciones, fundamentalmente, las privadas posean ventajas frente a sus oponentes, si desean aumentar su mercado, esto implica que cada día exige la búsqueda de la competitividad, para este objetivo, la generación de productividad de los trabajadores siempre ha sido una preocupación constante de los administradores para aumentar su radio de acción y lograr penetrar en los mercados. La productividad entendida como el rendimiento eficiencia de la actividad productiva de los hombres expresada por la correlación entre el gasto de trabajo (en escala de la sociedad, de una rama, de una empresa o de un solo trabajador) y la cantidad de bienes materiales producidos (establecida en dinero o en especie) en una unidad de tiempo (Alama, E. 2008).

En el contexto particular de las PYMES del Ecuador, según estudios realizados se ha determinado que una de sus principales debilidades es la falta de formalidad en las relaciones laborales con sus colaboradores lo que provoca una alta rotación del personal, el mismo que el momento que abandona la organización se lleva consigo un cúmulo de experiencias de aprendizaje personal que se conoce como conocimiento tácito, provocando con ello que la organización tenga que incurrir en nuevos costos de preparación, capacitación y desarrollo. El conocimiento es un recurso que las personas poseen y muchas veces no está sistematizado (Porter \& Millar, 1986), es decir no se ha logrado transformación de ese conocimiento tácito en conocimiento explícito lo que provoca que éste se quede solo en algunos individuos que lo poseen y no pase a ser propiedad de la organización quien es la que invierte en la capacitación y desarrollo de sus colaboradores, mediante la investigación se pretende conocer que influencia tienen los procesos de creación y transmisión del conocimiento en la productividad individual de los trabajadores a través de la implementación del modelos de Gestión del Conocimiento en las Pymes del sector textil de la ciudad de Riobamba.

A efectos de la presente investigación, la Gestión del Conocimiento se define como una estrategia organizacional que basada en un ambiente innovador y en el uso de las TIC, desarrolla

1 Master en Gerencia Empresarial MBA, Docente Titular de la Escuela Superior Politécnica de Chimborazo.carguello@espoch.edu.ec 
capacidades para: originar, almacenar, transferir, aplicar y proteger el conocimiento organizacional, con la finalidad de incrementar la competitividad y sustentabilidad de las organizaciones o empresas.

\section{METODOLOGÍA}

La presente es una Investigación Aplicada cuyo propósito es dar solución a situaciones o problemas concretos e identificables (Bunge, 1971), este tipo de investigación parte (por lo general, aunque no siempre) del conocimiento generado por la investigación básica, tanto para identificar problemas sobre los que se debe intervenir como para definir las estrategias de solución, el objetivo es predecir el comportamiento y desempeño de determinada acción, además es Explicativa porque define detalladamente las formas en las cuales el conocimiento se gestiona en la cadena productiva textil de las PYMES y cuáles son los factores y las magnitudes en que interaccionan desarrollando un modelo teórico (Hernández, Fernández y Baptista 2004), tiene un Diseño No Experimental en razón de que se realiza sin manipular deliberadamente variables. Se basa fundamentalmente en la observación de fenómenos tal y como se dan en su contexto natural para analizarlos con posterioridad, utiliza una Metodología Hipotética-Deductiva A continuación, se describen y explican los aspectos metodológicos y procedimentales generales de la investigación. Luego, los aspectos específicos de cada uno de los estudios empíricos efectuados y finalmente, los procedimientos y metodologías aplicadas para el diseño y validación del modelo de gestión del conocimiento (Arceo 2009) en la cadena de suministro de la industria textil.

\section{SISTEMA DE VARIABLES}

La Gestión del conocimiento es un constructo multidimensional (Alegre 2004) que se manifiesta a través diferentes características o capacidades organizativas. Así se puede considerar a la Gestión del conocimiento, como un modelo en el que las dimensiones representan el constructo, mientras los indicadores re presentan cada dimensión, siendo las dimensiones no observables. El constructo es una abstracción de orden superior a partir de las dimensiones (Buendía, Colás y Hernández 1998). Las dimensiones son en sí mismas constructos que se rigen como manifestaciones de un constructo más general. Por tanto, pueden tener sub dimensiones. Así el constructo general, en este caso la gestión del conocimiento, se puede representar como una integración de dimensio- nes, sub-dimensiones e indicadores (Lara, 2005). A efectos de la presente investigación, se diseñó un sistema de variables para estudiar el constructo gestión del conocimiento (variable independiente) y sus efectos sobre el constructo percepción de resultados (variable dependiente), en la cadena de suministro de la industria agroalimentaria, en cada uno de sus eslabones y en la cadena como un todo.

\subsection{Variable independiente: Gestión del conoci- miento}

La variable gestión del conocimiento se define como una estrategia que desarrolla capacidades para originar, almacenar, transferir, aplicar y proteger el conocimiento organizacional, con el fin de incrementar el capital intelectual de las cadenas de producción, en el marco del ciclo del conocimiento, en base a proyectos, necesidades y un ambiente innovador específico.

\subsection{Variable dependiente (Constructo): Percep- ción de resultados}

La variable dependiente, percepción de resultados, ha sido definida a efectos del presente estudio, como la formulación de juicios que sobre la realidad tienen las personas que integran las organizaciones, utilizando en este caso como referencia el Cuadro de Mando Integral CMI (Kaplan \& Norton, 1996) en sus dimensiones innovación, mejora de procesos internos, relaciones con el entorno y económica, a las cuales se adicionó una comparación referencial con empresas y trabajadores con funciones similares.

\subsection{Unidad de Análisis}

La investigación se realizó en la ciudad de Riobamba es la capital de la provincia de Chimborazo se encuentra ubicada en el centro del país, a $2.750 \mathrm{msnm}$. El tipo de empresas escogidas fueron las PYMES entendiéndose como el conjunto de pequeñas y medianas empresas que de acuerdo a su volumen de ventas, capital social, cantidad de trabajadores, y su nivel de producción o activos presentan características propias de este tipo de entidades económicas. El Sector de la Producción estudiado es el sector textil, utilizando como estrategia comprenderlo como un todo entendido para la investigación realizada como CADENA DE SUMINISTRO (Rice \& Hoppe 2001) y caracterizada por eslabones, estratos o clústeres, según la comprensión de los lectores, en el siguiente gráfico se pretende esquematizar lo expresado en forma literal. 


\subsection{Población y Muestra}

En la tabla 1 que se presenta a continuación se describe la composición de la población de estudio.

Tabla 1. Población

\begin{tabular}{|c|c|}
\hline Eslabón & Nro. De Establecimientos \\
\hline Proveedores de Materia Prima & 15 \\
\hline PYME Transformadora & 120 \\
\hline Comercio & 300 \\
\hline Total & $\mathbf{4 3 5}$ \\
\hline
\end{tabular}

Fuente: Elaboración propia

Datos: Cámara de la Pequeña Industria y Cámara de Comercio de Chimborazo

La tabla 2 resume la distribución de la muestra calculada con la fórmula para obtener la muestra de poblaciones finitas.

Tabla 2. Muestra

\begin{tabular}{|l|c|c|c|}
\hline \multicolumn{1}{|c|}{ Eslabón } & $\begin{array}{c}\text { Nro. De Esta- } \\
\text { blecimientos }\end{array}$ & $\begin{array}{c}\text { \% Correspon- } \\
\text { diente }\end{array}$ & Muestra \\
\hline $\begin{array}{l}\text { Proveedores } \\
\text { de Materia } \\
\text { Prima }\end{array}$ & 15 & 3,45 & 7 \\
\hline $\begin{array}{l}\text { PYME Trans- } \\
\text { formadora }\end{array}$ & 120 & 27,59 & 141 \\
\hline Comercio & 300 & 68,96 & 56 \\
\hline Total & $\mathbf{4 3 5}$ & $\mathbf{1 0 0}$ & $\mathbf{2 0 4}$ \\
\hline
\end{tabular}

Fuente: Elaboración propia

\subsection{Confiabilidad del instrumento}

Mediante el uso del programa estadístico informático SPSS, la tabla 3 resume el número de pruebas piloto que se corrieron para demostrar la confiabilidad del Instrumento.

Tabla 3. Resumen de procesamiento de casos

\begin{tabular}{|ll|r|r|}
\hline & $\mathrm{N}$ & \multicolumn{1}{|c|}{$\%$} \\
\hline Casos & Válido & 35 & 100,0 \\
& Excluido $^{\mathrm{a}}$ & 0 &, 0 \\
& Total & 35 & 100,0 \\
\hline
\end{tabular}

a. La eliminación por lista se basa en todas las variables del procedimiento.

Fuente: Elaboración propia
En la tabla 4 se puede observar los resultados obtenidos referente a los estadísticos de fiabilidad este caso el Alfa de Cronbach.

Tabla 4. Estadísticas de fiabilidad

\begin{tabular}{|r|r|}
\hline Alfa de Cronbach & N de elementos \\
\hline, 779 & 43 \\
\hline
\end{tabular}

Fuente: Elaboración propia

\subsection{Categorización de las Dimensiones}

Se planteó el Modelo Teórico de la investigación y la Variable Gestión del conocimiento se descompone en tres dimensiones: Gestión Estratégica del Conocimiento, Ambiente Innovador, Ciclo del Conocimiento o Gestión Funcional del Conocimiento (Afifi \&, Clark1990)

A continuación se presenta en las tablas 5 y 6 la categorización de cada una de las Variables tanto independiente y dependiente, respectivamente.

Tabla 5. Categorización de la Variable Independiente Gestión Del Conocimiento

\begin{tabular}{|ll|c|c|}
\hline & & Frecuencia & Porcentaje \\
\hline Válido & MALO & 32 & 15,7 \\
& REGULAR & 93 & 45,6 \\
& BUENO & 79 & 38,7 \\
& Total & 204 & 100,0 \\
\hline
\end{tabular}

Fuente: Elaboración propia

Tabla 6. Categorización de la Variable Dependiente Percepción De Resultados

\begin{tabular}{|ll|c|c|}
\hline & & Frecuencia & Porcentaje \\
\hline Válido & MALO & 35 & 17,2 \\
& REGULAR & 90 & 44,1 \\
& BUENO & 79 & 38,7 \\
& Total & 204 & 100,0 \\
\hline
\end{tabular}

Fuente: Elaboración propia

\subsection{Modelo de Gestión del Conocimiento}

A continuación se ha diseñado el Modelo de Gestión del conocimiento para las PYMES del sector Textil de la ciudad de Riobamba, basándose sobre la base de los resultados y análisis de los estudios empíricos tratados en el capítulo anterior. 


\subsection{Selección y análisis de los indicadores de- terminantes}

Dentro de la metodología del estudio se realizó en su primera fase, el diseño, validación y aplicación del instrumento de medición (encuesta) que se aplicó a través de entrevistas estructuradas, en las cuales la Variable Gestión del Conocimiento fue observada a través de indicadores medidos por la escala de Lickert (Campo-Arias, A y Oviedo, H 2008). Los instrumentos de medición cumplieron con las pruebas de validez y fiabilidad.

Luego para determinar los indicadores válidos y fiables se procedió a determinar la media, la moda y la frecuencia relativa para cada uno de los indicadores de cada una de las subdimensiones y dimensiones, considerando como frecuencias relativas a la suma de los valores de las frecuencias individuales de las escalas superiores de Likert (Moderadamente de acuerdo y Totalmente de acuerdo) con el fin de obtener un valor significativo para la elaboración del modelo, puesto que a criterio del investigador existe la voluntad manifiesta del informante en estar de acuerdo con las preposiciones consultadas.

En la tabla 7 resumen que se presenta a continuación se puede apreciar que los indicadores de mayor Frecuencia Relativa para cada una de las dimensiones.

Una vez que se ha obtenido los indicadores con mayor frecuencia relativa se cuenta con los insumos necesarios para el diseño del Modelo de Gestión del conocimiento para el sector en estudio a continuación la tabla 7 que resume lo anteriormente manifestado.

Tabla 7. Cuadro Resumen Indicadores válidos y fiables de la gestión del conocimiento Todas las Dimensión

\begin{tabular}{|c|c|c|c|c|c|}
\hline Dimensión & Subdimensión & Indicador & Mediana & Moda & $\begin{array}{l}\text { Frecuencia } \\
\text { Relativa \% }\end{array}$ \\
\hline \multirow{4}{*}{$\begin{array}{l}\text { Gestión Estratégica } \\
\text { del Conocimiento }\end{array}$} & \multirow{2}{*}{$\begin{array}{l}\text { Estrategias de } \\
\quad \text { la GC }\end{array}$} & $\begin{array}{l}\text { Enfasis en cuantificar y manejar el capital intangible } \\
\text { (Nombres, marcas, prestigio, relaciones con clientes, etc.) }\end{array}$ & 4 & 4 & 91,7 \\
\hline & & $\begin{array}{l}\text { Enfasis en la innovación y producción de nuevo } \\
\text { conocimiento aplicado en la empresa }\end{array}$ & 4 & 5 & 86,3 \\
\hline & \multirow{2}{*}{$\begin{array}{l}\text { Objetivos de la } \\
\text { GC }\end{array}$} & Mejorar la calidad de los productos y servicios. & 4 & 4 & 76 \\
\hline & & Reducir costos & 4 & 4 & 63,2 \\
\hline \multirow[b]{2}{*}{ Ambiente Innovador } & $\begin{array}{l}\text { Cultura de } \\
\text { Innovación }\end{array}$ & $\begin{array}{l}\text { 6.- Equipamiento y procedimientos innovadores que se } \\
\text { apoyan en el uso del computador y el internet. }\end{array}$ & 5 & 5 & 89,3 \\
\hline & $\begin{array}{l}\text { Liderazgo } \\
\text { Innovador }\end{array}$ & $\begin{array}{l}\text { 1.- Los dueños, directivos y supervisores, estimulan con } \\
\text { su liderazgo la innovación. }\end{array}$ & 4 & 4 & 74 \\
\hline \multirow{10}{*}{$\begin{array}{l}\text { Ciclo del } \\
\text { Conocimiento o } \\
\text { Gestión Funcional } \\
\text { del GC }\end{array}$} & Origen & $\begin{array}{l}\text { De la adquisición del conocimiento en cualquiera de sus } \\
\text { formas (equipos, asesorías, literatura especializada, etc). }\end{array}$ & 4 & 5 & 86,8 \\
\hline & & $\begin{array}{l}\text { De la acción de la propia empresa, con estrategia orga- } \\
\text { nizacional a la innovación y mejora de procesos y pro- } \\
\text { ductos. }\end{array}$ & 4 & 4 & 72,5 \\
\hline & Almacenamiento & $\begin{array}{l}\text { En forma tácita: en la memoria de los trabajadores, rutinas } \\
\text { laborales, la cultura organizacional, las tradiciones. }\end{array}$ & 4 & 3 & 52,9 \\
\hline & & $\begin{array}{l}\text { En forma explícita en documentos de papel: informes, } \\
\text { normas y procedimientos, manuales de operación y man- } \\
\text { tenimiento, manuales de calidad, que son consultados } \\
\text { físicamente. }\end{array}$ & 3 & 3 & 40,2 \\
\hline & Transferencia & $\begin{array}{l}\text { En forma tácita por acción de los trabajadores, superviso- } \\
\text { res y/o directivos, a través de intercambio de conocimien- } \\
\text { tos y experiencias de manera verbal o por imitación. }\end{array}$ & 4 & 4 & 78,4 \\
\hline & & $\begin{array}{l}\text { En forma tácita por acción de la gestión de la empresa, } \\
\text { que realiza acciones tales como: rotación del personal, } \\
\text { asignando a personal de mayor experiencia y formación. }\end{array}$ & 4 & 4 & 61,3 \\
\hline & Aplicación & $\begin{array}{l}\text { En equipo; coordinando su trabajo con el de sus compa- } \\
\text { ñeros en función del cumplimento de los objetivos defini- } \\
\text { dos por la empresa. }\end{array}$ & 4 & 4 & 93,1 \\
\hline & & $\begin{array}{l}\text { En forma rutinaria; los procedimientos y actividades se } \\
\text { realizan prácticamente en ausencia de reglas, normativas } \\
\text { o incluso comunicación verbal. }\end{array}$ & 4 & 4 & 77 \\
\hline & Protección & $\begin{array}{l}\text { Obteniendo el reconocimiento por parte de los clientes de } \\
\text { una calidad superior y/o especial, debido a las caracterís- } \\
\text { ticas propias de los productos y el proceso. }\end{array}$ & 4 & 4 & 61,3 \\
\hline & & $\begin{array}{l}\text { Actuando como vanguardia en las innovaciones desarrolladas } \\
\text { en la empresa, lo cual le ofrece ventajas sobre sus competidores } \\
\text { y mayor aceptación por parte de los clientes. }\end{array}$ & 4 & 4 & 54,5 \\
\hline
\end{tabular}

Fuente: Elaboración propia 
Seleccionados y analizados los indicadores determinantes, se hace oportuno examinar las relaciones que existen entre ellos. Por tanto, se ha procedido a realizar una representación esquemática del conjunto de conceptos estudiados que permiten diseñar el Modelo propuesto.

En la figura 1, se puede observar la relación existente entre la cadena de suministro de la PYME, la gestión del conocimiento y la percepción de resultados y el Ciclo del Conocimiento. En el mismo, se identifica los tres eslabones de la cadena de suministro Proveedores, PYME transformadora y Co- mercio el sentido ascendente de la cadena expresan los incrementos y mejora de la percepción de resultados en el manejo de un Ambiente Innovador que cuente con el equipamiento y contexto necesario que apoye la generación de innovaciones y posean directivos que estimulen este propósito.

Mencionados incrementos serán posibles solo si existe lo siguiente:

1. Una estrategia definida que haga énfasis en el capital intelectual cumpla con el ciclo del conocimiento o su gestión funcional.

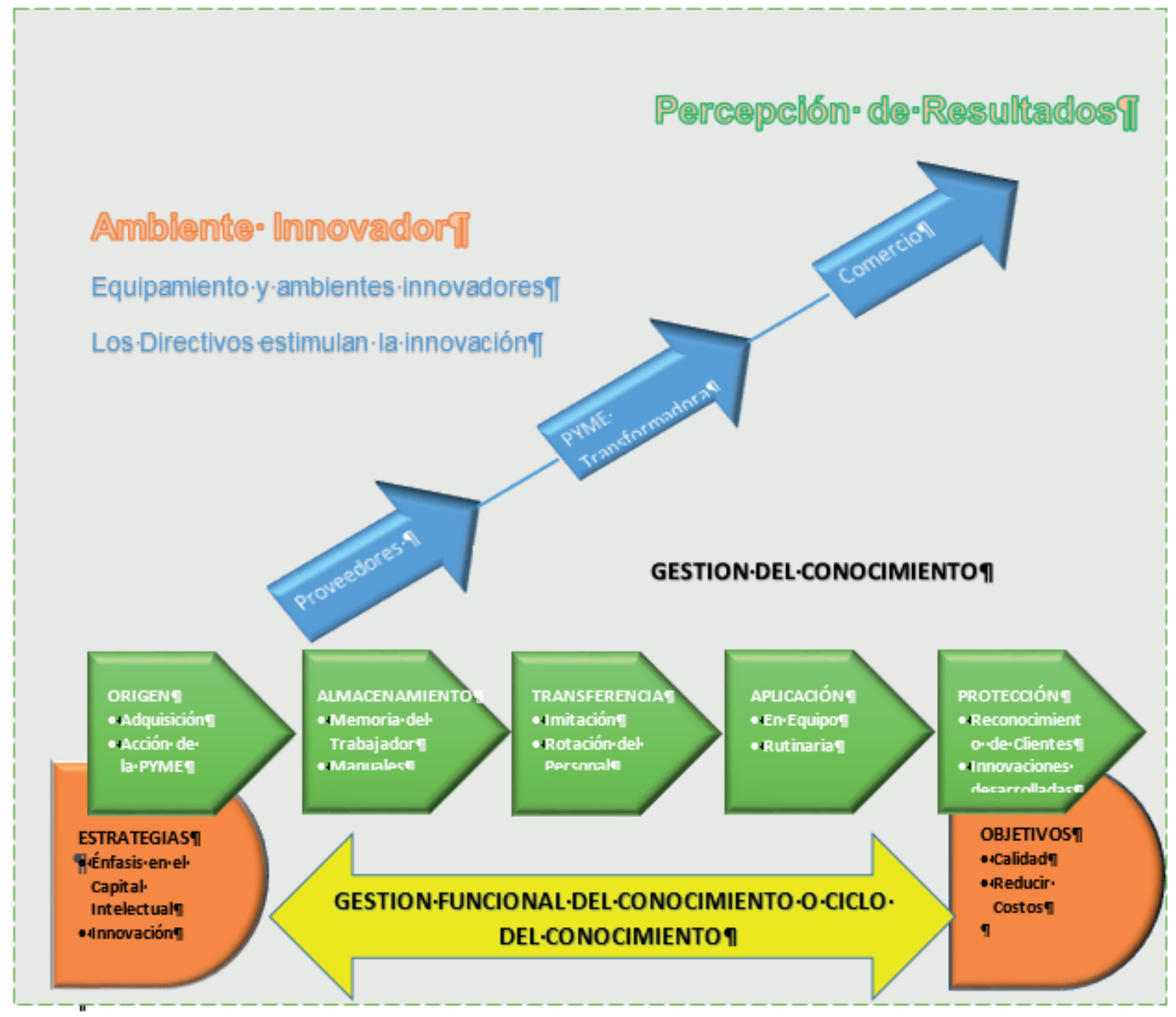

Figura 1. Diseño del esquema representativo del Modelo Teórico de la Gestión del conocimiento Fuente: Elaboración propia

2. Con el origen del conocimiento que privilegie la adquisición del mismo en cualquiera de sus formas (equipos, asesorías, literatura especializada, etc), a través de la acción de la propia empresa, con estrategia organizacional orientada hacia la innovación y mejora de procesos y productos.
3. Almacenamiento del conocimiento, en forma tácita es decir en la memoria de los trabajadores, rutinas laborales, la cultura organizacional, las tradiciones y en forma explícita en documentos de papel: del conocimiento informes, normas y procedimientos, manuales de operación y mantenimiento, manuales de calidad, que son consultados físicamente. 
4. Transferencia del conocimiento en forma tácita por acción de los trabajadores, supervisores y/o directivos, a través de la comunicación y el intercambio de conocimientos y experiencias de manera verbal o por imitación, entre los trabajadores de todos los niveles jerárquicos y funcionales de la empresa y por la gestión de la empresa, que realiza acciones tales como: rotación del personal, asignando a personal de mayor experiencia y formación trabajadores que se están entrenando, otras tácticas organizacionales para transferir el conocimiento.

5. Aplicación del conocimiento en equipo; coordinando su trabajo con el de sus compañeros en función del cumplimento de los objetivos definidos por la empresa, considerando acciones que se realicen en forma rutinaria con los procedimientos y actividades que se realizan prácticamente en ausencia de reglas, normativas o incluso comunicación verbal.

6. Protección del conocimiento, la mejor manera de protegerlo es obteniendo el reconocimiento por parte de los clientes de una calidad superior y/o especial, debido a las características propias de los productos y el proceso empresarial en el que se desempeña, actuando como vanguardia en las innovaciones desarrolladas en la empresa, lo cual le ofrece ventajas sobre sus competidores y mayor aceptación por parte de los clientes.

7. Objetivos que cumplan sus características básicas a entender deben ser claros, precisos y realizables y además busquen mejorar la calidad de los productos y servicios y la reducción de costos.

El modelo diseñado tiene el carácter de sistemático es decir existes relaciones directas entre sus dimensiones y subdimensiones no se puede prescindir de ninguna de ellas, además se retroalimenta de manera constante, en razón de que una vez cumplido el objetivo automáticamente se genera nuevo conocimiento y se plantean nuevas estrategias. A continuación se presenta el esquema sobre las relaciones entre las distintas subdimensiones y las prácticas de la Gestión del Conocimiento, determinando también la secuencia de ocurrencia de las mismas.

El flujo de conocimiento que se origina en el sistema y que tiene impacto válido, fiable y consistente sobre la gestión del conocimiento y esta sobre percepción de resultados se almacena, la memoria de los trabajadores se traslada en documentos de papel que son archivados de manera organizada y adecuada, con fácil acceso a su consulta (Manuales), se transfieren a través de la imitación de las actividades generadas mediante la rotación del personal, buscando la participación de equipos para fortalecer labores rutinarias que generan valor y se visibilizan en innovaciones que son reconocidas por los clientes.

La figura 2, que se presenta a continuación expresa las relaciones entre subdimensiones y prácticas de la Gestión del Conocimiento.

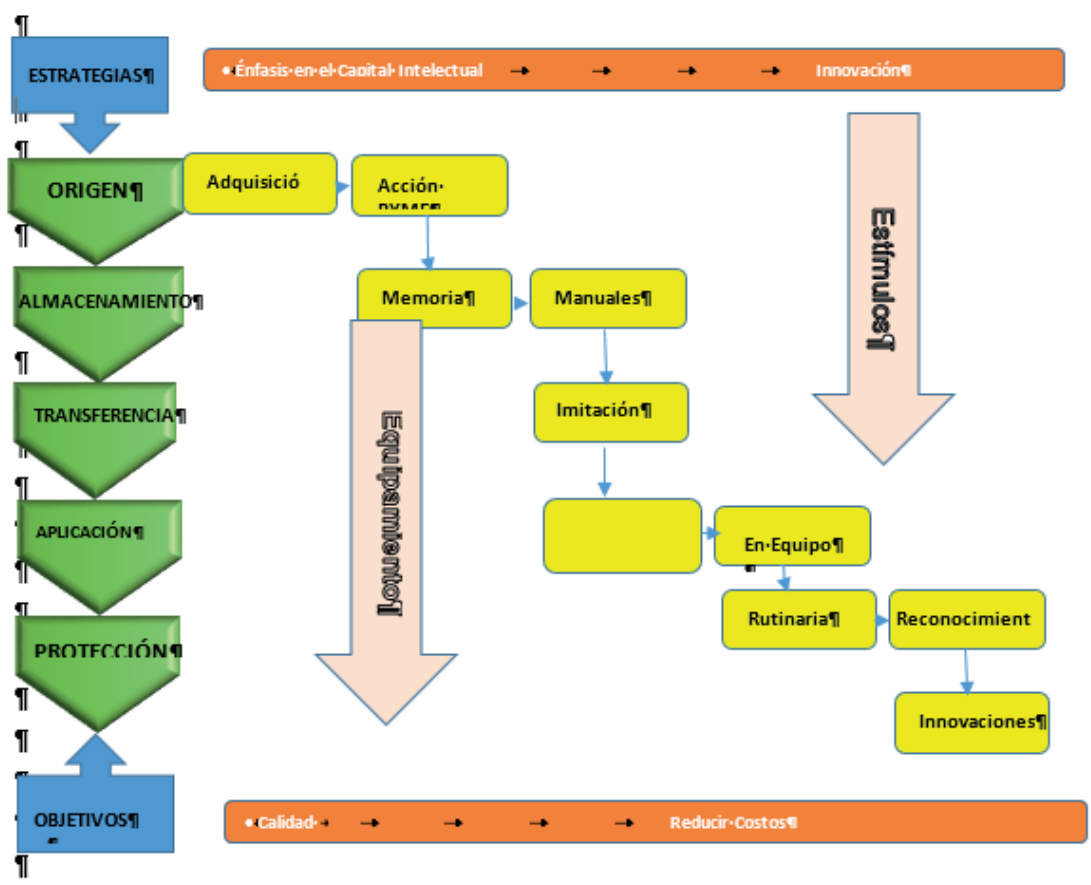

Figura 2. Relaciones entre Subdimensiones y prácticas de la GC

Fuente: Elaboración propia 


\section{CONCLUSIÓN GENERAL}

El Modelo de Gestión del Conocimiento diseñado permite establecer que existe una influencia significativa en la Percepción de Resultados de las PYMES del sector textil de la ciudad de Riobamba, este efecto fue verificado en cada eslabón y en la cadena de suministro del sector textil de la ciudad de Riobamba con un alto grado de validez, fiabilidad y consistencia, en la prueba estadística un chi-cuadrado de 302,729 con un $\mathrm{P}$-valor $=0.000$, el cual es menor a 0.05 el nivel de significancia, por lo tanto se rechaza la hipótesis nula y se acepta la alternante, demostrando que existe evidencia estadística para afirmar que el desarrollo del Modelo de Gestión del Conocimiento influye significativamente en la Percepción de Resultados de las PYMES del sector textil de la ciudad de Riobamba.

\section{CONCLUSIONES ESPECÍFICAS}

- La dimensión gestión estratégica del conocimiento influye significativamente en la percepción de los resultados de las PYMES del sector textil de la ciudad de Riobamba, en la prueba estadística un chi-cuadrado de 91,498 con un P-valor $=0.000$, el cual es menor a 0.05 el nivel de significancia, por lo tanto se rechaza la hipótesis nula y se acepta la alternante, se demuestra que existe evidencia estadística para afirmar que el desarrollo de la dimensión gestión estratégica del conocimiento influye significativamente en la percepción de los resultados de las PYMES del sector textil de la ciudad de Riobamba. Además, se puede apreciar que los indicadores de mayor Frecuencia Relativa en los componentes o subdimensiones de la Dimensión Gestión estratégica del conocimiento fueron para la Subdimensión Estrategias de la Gestión del Conocimiento En primer lugar el indicador "Énfasis en cuantificar y manejar el capital intangible (Nombres, marcas, prestigio, relaciones con clientes, etc.)", con una Media de 4,37, una Moda de 4 y una Frecuencia Relativa del 91,7\%.En segundo lugar el indicador "Énfasis en la innovación y producción de nuevo conocimiento aplicado en la empresa", con una Media de 4,32, una Moda de 5 y Frecuencia Relativa de $86,3 \%$; y para la Subdimensión Objetivos de la Gestión del Conocimiento en primer lugar el indicador "Mejorar la calidad de los productos y servicios.", con una Media de 3,98, una Moda de 4 y una Frecuencia Relativa del $76 \%$.En segundo lugar el indicador "Reducir costos", con una Media de 3,71, una Moda de 4 y Frecuencia Relativa de 63,2\%.
- El Ambiente Innovador influye significativamente en la percepción de los resultados de las PYMES del sector textil de la ciudad de Riobamba, esto se afirma en razón de que en la prueba estadística un chi-cuadrado de 36,548 con un P-valor $=0.000$, el cual es menor a 0.05 el nivel de significancia, por lo tanto se rechazó la hipótesis nula y se aceptó la alternante, se demuestra que existe evidencia estadística para afirmar que a dimensión Ambiente Innovador influye significativamente en la percepción de los resultados de las PYMES del sector textil de la ciudad de Riobamba; los indicadores con mayores estadísticos descriptivos fueron en primer lugar el indicador "Equipamiento y procedimientos innovadores que se apoyan en el uso del computador y el internet", perteneciente a la Subdimensión Cultura de Innovación, con una Media de 4,45, una Moda de 5 y 89,3\% de Frecuencia Relativa, y en segundo lugar el indicador "Los dueños, directivos y supervisores, estimulan con su liderazgo la innovación.", perteneciente a la Subdimensión Liderazgo Innovador, con una Media de 3,36, una Moda de 4 y 74\% de Frecuencia Relativa.

- La dimensión del ciclo del conocimiento influye significativamente en la percepción de los resultados de las PYMES del sector textil de la ciudad de Riobamba esta afirmación se la hace en consecuencia de que luego de realizada la prueba estadística se obtuvo un chi-cuadrado de 277,231 con un P-valor $=0.000$, el cual es menor a 0.05 el nivel de significancia, por esta razón se rechazó la hipótesis nula y se aceptó la alternante, existiendo evidencia estadística para afirmar que el desarrollo del Modelo de Gestión del Conocimiento en la dimensión del ciclo del conocimiento influye significativamente en la percepción de los resultados de las PYMES del sector textil de la ciudad de Riobamba.

\section{REFERENCIAS BIBLIOGRÁFICAS}

[1] Afifi \& Clark, (1990) Computer-aided. Multivariate Analysis. 2a. ed. New York-USA: Van Nostrand Reinholds.

[2] Alama, E. (2008) Capital intelectual y resultados empresariales en las empresas de servicios profesionales de España. Tesis Doctoral Departamento de Organización de Empresas, Facultad de Ciencias Económicas y Empresariales, Madrid: Universidad Complutense de Madrid. 
[3] Alegre, J. (2004) La gestión del conocimiento como motor de la innovación. Lecciones de la industria de alta tecnología para la empresa. Castellón- España: Universitat Jaume I. Servei de Comuniació i Publicacions.

[4] Araque, W. (2000) Asistencia Integral: herramienta para mejorar la competitividad de las PyME. Desafíos No.4. Quito Ecuador: Cámara de la Pequeña Industria de Pichincha.

[5] Arceo, G. (2009) El impacto de la gestión del conocimiento y las tecnologías de información en la innovación: un estudio en las PYMEs del sector agroalimentario de Cataluña. Tesis Doctoral en Administración y Dirección de Empresas. Cataluña-España: Departamento de Organización de Empresas. Universidad Politécnica de Cataluya.

[6] Buendía, L., Colás, P. y Hernández, F. (1998) Métodos de Investigación en Psicopedagogía. Madrid: McGraw-Hill.

[7] Bunge, M. (1971) La Ciencia. Su método y Filosofía. 4a. ed. Buenos Aires Argentina: Editorial Universitaria de Buenos Aires.

[8] Campo-Arias, A y Oviedo, H C; (2008). Propiedades Psicométricas de una Escala: la Consistencia Interna. Revista de Salud Pública,
Recuperado de http://www.redalyc.org/articulo. oa?id=42210515

[9] Hernández, R., Fernández, C., Baptista, L. (2004) Metodología de la Investigación. México. DF.: Mc Graw Hill Interamericana.

[10] Kaplan, R., Norton, D. (1996) Cuadro de mando integral. Barcelona.: Gestión 2000. Barcelona.

[11] Kaplan, R., Norton, D. (2004) Mapas estratégicos. Convirtiendo los activos intangibles en resultados tangibles. Barcelona: Gestión 2000.

[12] Lara, F. (2005) Estudio de la gestión del conocimiento sobre los resultados organizativos: Análisis del efecto mediador de las competencias directivas. Valencia España: Departamento de organización de empresas, economía financiera y contabilidad. Tesis Doctoral. Universidad Politécnica de Valencia.

[13] Porter, M., Millar, V. (1986) Cómo obtener ventajas competitivas por medio de la Información. Boston-USA: Harvard-Deusto Business Review.

[14] Rice, Jr., Hoppe, R. (2001) Supply chain versus supply chain: The hype and the reality. Supply Chain Management Review http://web.mit.edu/ supplychain/repository/scvssc.pdf 